\title{
Carbon Nanoparticles-Decorated Carbon Nanotubes
}

\author{
Ahmed Awadallah-F \& Shaheen Al-Muhtaseb (ib) \\ Multi walled carbon nanotubes (MWCNTs) were decorated by activated carbon nanoparticles of \\ resorcinol-formaldehyde aerogels. Carbon nanospheres and MWCNTs were mixed by equal mass ratios \\ for different durations. The products were characterized by Raman spectroscopy, thermal gravimetric \\ analysis, nanoscanning electron microscopy, transmission electron microscopy and x-ray diffraction. \\ The results indicated that a significant decoration with carbon nanoparticles occurred onto the \\ MWCNTs.
}

Carbon nanotubes (CNTs) drew a remarkable attention from their first discovery. This is attributed to their unique features, such as mechanical properties, electric properties, thermal stability, high chemical resistance and large surface areas ${ }^{1}$. As a result of these characteristics, they became strong candidates for numerous applications; including catalytic processes, water treatment, drug delivery, gene transfer, transparent conducting membranes and electrochemical analysis ${ }^{2-7}$. The idea of $\mathrm{CNT}$ decoration was introduced to widen their applications in different fields. It was reported in various works of literature that it was possible to decorate CNTs with either organic compounds or metallic nanoparticles ${ }^{8-10}$. The importance of carbon aerogels is assigned to their hierarchical porous properties, which makes them suitable for use in numerous applications ${ }^{11-14}$. For example, carbon aerogels are utilized in catalyst support, separation tools, eclectic supercapacitor materials and battery construction ${ }^{15,16}$. It is noteworthy to mention that, to the authors' knowledge, the carbon-carbon decoration of multiwall carbon nanotubes (MWCNTs) by carbon nanoparticles (CNp) was not tackled in literature. Therefore, in this work, we disclose our method of decorating MWCNTs with resorcinol-formaldehyde activated carbon aerogel (RFA) CNp. The hybrid carbon nanoproducts will be characterized by various devices for tracking the changes of outcome carbon nanoproducts.

\section{Results and Discussion}

Figure $1(\mathrm{a}-\mathrm{c})$ exibits the characterestics of MWCNTs and RFA-CNp via XRD, Raman spectra and TGA, respectively. Figure 1a exposes the XRD patterns of MWCNTs (black line) and RFA-CNp (red line) samples. It was observed from Fig. 1 a that the characteristic bands of MWCNTs are at $2 \theta=26^{\circ}, 42^{\circ}$ and $53.8^{\circ}$; which refer to (002), (100) and (004) reflection of the MWNTs, accdringly ${ }^{17}$. Moreover, RFA-CNp has no crystalline peaks as seen from Fig. 1a. The higher crystallinity in MWCNTs than RFA-CNp is due to the graphitic portion that results in the high order of MWCNTs' structures.

Figure $1 \mathrm{~b}$ shows the Raman spectra of MWCNTs (black line) and RFA-CNp (red line) samples. It is noteworthy to declare that the Raman spectra can be utilized to estimate the degree of defects or disorders of MWCNTs and RFA-CNp. The three fingerprint bands of pristine MWCNTs are the D-band at $1314 \mathrm{~cm}^{-1}$, G-band at $1576 \mathrm{~cm}^{-1}$ and G'-band at $2625 \mathrm{~cm}^{-1}$. The intensity of the D-band $\left(\mathrm{I}_{\mathrm{D}}\right)$ indicates to disordered or amorphous carbon portions, while that of the $\mathrm{G}$ band $\left(\mathrm{I}_{\mathrm{G}}\right)$ indicates to graphite or ordered carbon portions in the MWCNTs, and that of the $\mathrm{G}^{\prime}$ band indicates to a second harmonic of the D-band ${ }^{18}$. Moreover, the relative crystallinity can be indicated by the $\mathrm{I}_{\mathrm{D}} / \mathrm{I}_{\mathrm{G}}$ ratio. The values of $\mathrm{I}_{\mathrm{D}} / \mathrm{I}_{\mathrm{G}}$ ratio are 0.48 and 1.25 for MWCNTs and RFA-CNp, respectively. Thus, the order (crystallinity) of MWCNTs is higher than that of RFA-CNp. Figure 1c shows the TGA thermograms of MWCNTs (black line) and RFA-CNp (red line) samples. It is observed that the thermal stability of MWCNTs is higher than that of RFA-CNp. This is attributed to the presence of graphic structure that resists the thermal decomposition in MWCNTs, on contrary to the amorphous structure that has less resistance to thermal decomposition in RFA-CNp.

Figure 2(a-c) exibits the characterestics of MWCNTs decorated with RFA-CNp via XRD, Raman spectra and TGA, accordingly; for samples decorated for various number of days as denoted by the sample codes in the form $n \mathrm{D}$ where the number $n$ stands for the number of days in mixing. It is seen form the XRD patterns in Fig. 2a that the crystallinity of MWCNTs decreases by increasing the time of mixture stirring. The characteristic peaks of MWCNTs are at $2 \theta=26^{\circ}, 42^{\circ}$ and $53.8^{\circ}$; which refer to the (002), (100) and (004) reflections of the MWCNTs, 

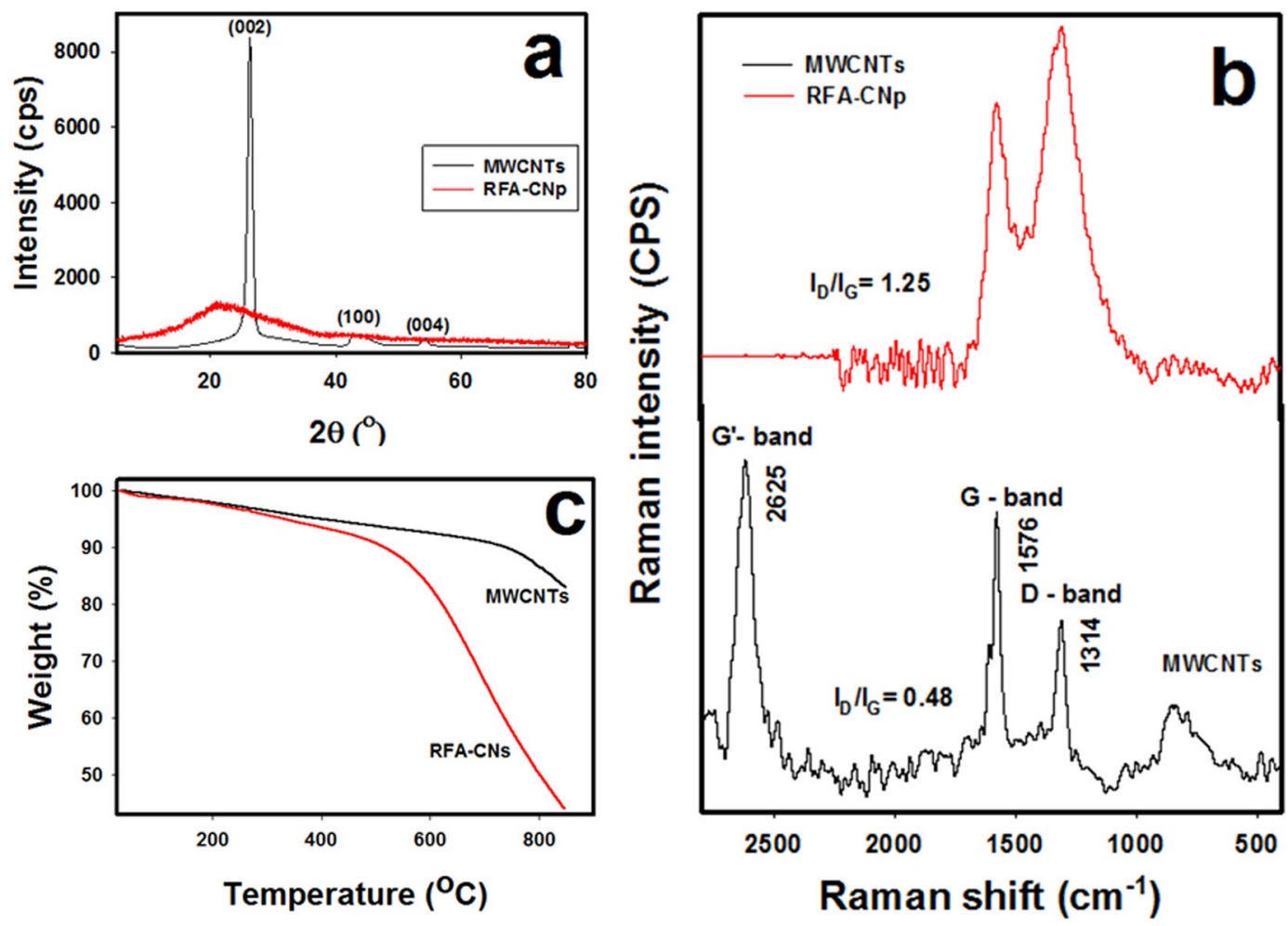

Figure 1. (a) XRD patterns, (b) Raman spectra and (c) TGA thermograms of MWCNTs (black line) RFA-CNp (red line) samples.
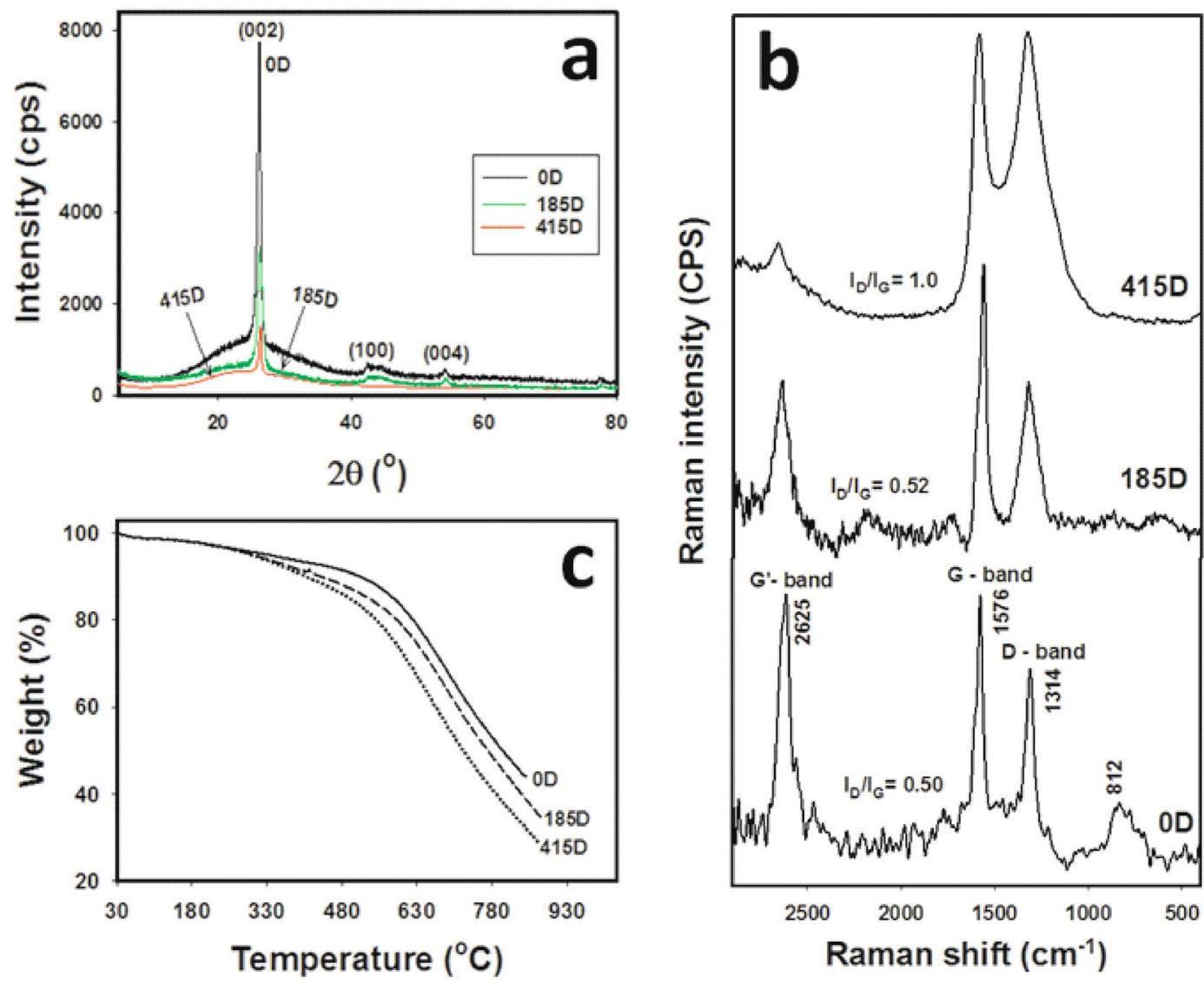

Figure 2. (a) XRD patterns, (b) Raman spectra and (c) TGA thermograms of MWCNTs/RFA-CNp hybrids with $50 / 50 \mathrm{~g} / \mathrm{g}$ at mixing times of 0,185 and 415 days. 
respectively ${ }^{19}$. The peak observed at $26^{\circ}$ indicates to the (002) diffractions of graphite, which confirms that the hexagonal graphite structure is still in a good state in all samples ${ }^{20}$. The peaks at $2 \theta$ of $42.4^{\circ}$ and $53.8^{\circ}$ refer to the in-plane graphitic structure ${ }^{21}$. Furthermore, the intensities of the $(002)$ diffraction in the samples $0 \mathrm{D}, 185 \mathrm{D}$ and $415 \mathrm{D}$ are 7774,3229 and 1596, accordingly. Hence, the intensity of the (002) diffraction of 185D represents $41.53 \%$ of that of $0 \mathrm{D}$ and the intensity of $415 \mathrm{D}$ represents $20.52 \%$ of that of $0 \mathrm{D}$. Figure $2 \mathrm{~b}$ shows the Raman spectra of MWCNTs/RFA-CNp hybrids. Raman spectra can be utilized to assess the degree of defects or disorders of MWCNTs after decoration with RFA-CNp. Three characteristic peaks of pristine MWCNTs can be seen as the $\mathrm{D}$-band at $1314 \mathrm{~cm}^{-1}, \mathrm{G}$-band at $1576 \mathrm{~cm}^{-1}$ and $\mathrm{G}^{\prime}$-band at $2625 \mathrm{~cm}^{-1}$. The intensity of the $\mathrm{D}$ band $\left(\mathrm{I}_{\mathrm{D}}\right)$ indicates to disordered or amorphous carbon portions, while that of the $\mathrm{G}$ band $\left(\mathrm{I}_{\mathrm{G}}\right)$ refers to graphite or ordered carbon portions in the MWCNTs, and that of the G' band indicates to a second harmonic of the D-line ${ }^{22}$. Moreover, the disorder/defects can be determined by the $\mathrm{I}_{\mathrm{D}} / \mathrm{I}_{\mathrm{G}}$ ratio. The values of $\mathrm{I}_{\mathrm{D}} / \mathrm{I}_{\mathrm{G}}$ ratio are $0.50,0.52$ and 1.00 for the $0 \mathrm{D}$, $185 \mathrm{D}$ and 415D samples, accordingly. Therefore, the degree of disorder/defects of decorated CNTs decreases when increasing the mixing time. On other words, the more migration of RFA-CNp particles to the surface of MWCNTs, the disorder/defects phenomena increases.

Figure 2c exposes TGA thermograms of MWCNTs/RFA-CNp mixture. It was observed that, when increasing the mixing time, the thermal stability of MWCNTs decreases. For instance, the mass losses of the 0D, 18D5 and $415 \mathrm{D}$ samples at $353^{\circ} \mathrm{C}$ were $5.11,6.56$ and $7.38 \%$, respectively. Furthermore, the mass losses of 0D, 185D and $415 \mathrm{D}$ samples at $484^{\circ} \mathrm{C}$ were $8.67,11.93$ and $13.73 \%$, respectively. The mass losses (\%) of 0,185 and $415 \mathrm{D}$ at $678^{\circ} \mathrm{C}$ were $29.17,34.23$ and $42.69 \%$, respectively. Similarly, the mass losses of $0 \mathrm{D}, 185 \mathrm{D}$ and $415 \mathrm{D}$ at $842^{\circ} \mathrm{C}$ were 55.52, 61.71 and $69.02 \%$, respectively. Consequently, the presence of RFA-CNp into the matrix of MWCNTs impacts significantly the samples' thermal stability. The migration of RFA-CNp to the surface of MWCNTs surface weakens the graphic structure of MWCNTs. XRD, Raman spectra and TGA of the utilized MWCNTs and RFA-CNp are described in the Fig. 1.

Figure 3(a-h) exhibits the NanoSEM photomicrographs of MWCNTs/RFA-CNp at variable times of stirring; 0D, 185D and 415D. Figure 3(a,b) exposes the NanoSEM photomicrographs of MWCNTs and RFA-CNp samples, respectively. It is seen from Fig. 3a that the morphology of MWCNTs is tubular in shape. From Fig. 3b, it is observed that the morphology of RFA-CNp seems like a spherical style. On the other hand, Fig. 3(c,d) exposes the two amplifications of MWCNTs/RFA-CNp at 0D. It can be noticed that the morphology MWCNTs are in good state and that of RFA-CNp also remains well. Figure 3(e,f) shows the two amplifications of MWCNTs/RFA-CNp of $185 \mathrm{D}$ at $1 \mu \mathrm{m}$ and $500 \mathrm{~nm}$, accordingly. It can be observed from Fig. 3(e,f) that the change appeared clearly on the outer surface morphology of MWCNTs, which becomes decorated with RFA-CNp. Figure 3(g,h) shows the two amplifications of MWCNTs/RFA-CNp of $415 \mathrm{D}$ at $1 \mu \mathrm{m}$ and $500 \mathrm{~nm}$, accordingly. It can be noticed that the attachment RFA-CNp onto MWCNTs grows more densely and abundantly in the case of 415D. Therefore, it can be deduced that this hybrid carbon product has new features could be used in versatile applications and need further investigations.

Figure 4(a-f) exposes the TEM photomicrographs of MWCNTs/RFA-CNp at variable times of stirring; 0D, 185D and 415D. Figure 4(a,b) shows the TEM photomicrographs of MWCNTs and RFA-CNs samples, accordingly. It can be confirmed again that the morphological shapes of MWCNTs and RFA-CNp are tubular and seemingly spherical style, accordingly. Figure $4(\mathrm{c}, \mathrm{d})$ shows the MWCNTs/RFA-CNp of $0 \mathrm{D}$ at two magnifications; $1 \mu \mathrm{m}$ and $500 \mathrm{~nm}$, respectively. It is observed from both magnifications that the morphologies of both of the MWCNTs and RFA-CNp remain well intact. Figure 4(e,f) exhibits the MWCNTs/RFA-CNs after stirring of 185D at two magnifications; $1 \mu \mathrm{m}$ and $500 \mathrm{~nm}$, respectively. Through the observation it can be deduced that the MWCNTs become decorated with RFA-CNp. Figure 4(g,h) shows the MWCNTs/RFA-CNp of 415D at two magnifications; $1 \mu \mathrm{m}$ and $500 \mathrm{~nm}$, respectively. It can be noticed from both photos that the MWCNTs become decorated densely and abundantly with RFA-CNp. Overall observation, the presence of RFA-CNp onto the surface of MWCNTs led to new decorated carbon-carbon products with different features than the pristine MWCNTs or RFA-CNp.

\section{Conclusion}

The carbon-carbon decoration, specifically MWCNTs with carbon nanoparticles, is a novel trend that can lead to expanding the potential applications of such nanomaterials. This work is just a trial to confirm that it is possible to decorate MWCNTs with carbon aerogel nanoparticles (RFA-CNp) as an example. This is done by mixing in methanol for long durations at a relatively high temperature. Various characterization techniques; including XRD, Raman spectra, TGA, NanoSEM and TEM were utilized to investigate the decorated products. The results referred to significant changes in the properties of pristine MWCNTs and RFA-CNp, which confirmed the formation of hybrid carbon nanoproducts. Moreover, in comparison to the pristine MWCNTs or RFA-CNp, the produced hybrid carbon nanoproducts are featured with unique morphologies, crystallinities and extents of order/defects in their structures. Overall, it was found that mixing for 185 days resulted in decorating the MWCNTs with RFA-CNp to a noticeable extent, whereas mixing for 415 days resulted in a significant (and in an abundance of) decoration. These hybrid carbon nanoproducts could be used in numerous applications and are worthy of further investigations.

\section{Experimental section}

Materials. MWCNTs (purity $>90 \%$, with a diameter of $110-170 \mathrm{~nm}$ and length of $5-9 \mu \mathrm{m}$, resorcinol (purity $99 \%$ ) and formaldehyde (containing 10-15\% methanol as stabilizer, 37 wt.\% in $\mathrm{H}_{2} \mathrm{O}$ ) were purchased from Sigma-Aldrich, (Germany). Sodium carbonate anhydrous (Fisher Scientific, UK). Ultra-pure water supplied from Elix ${ }^{\circledR} 70$ Water Purification System from Millipore Sigma. Other reagents (Acetone, methanol, acetic acid, nitric acid and ammonium hydroxide) are of analytical reagent grade. All chemicals are utilized as-received. 

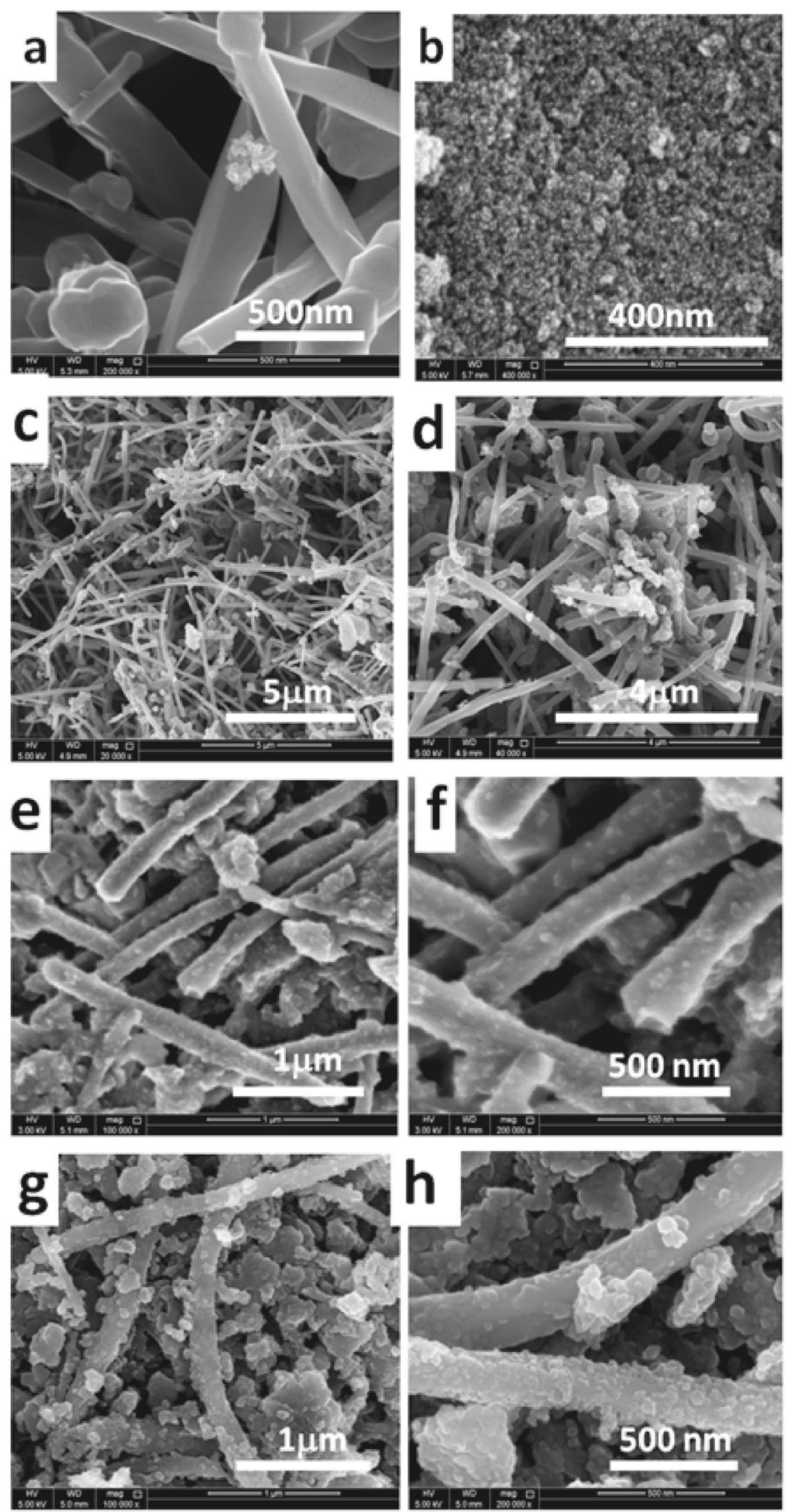

Figure 3. NanoSEM photomicrographs of MWCNTs/RFA-CNp with 50/50 g/g mixing ratio at different mixing times and different scales of magnifications. (a,b) refers to pristine MWCTs and CNp, respectively. Each couple of photomicrographs of (c,d) 0D, (e,f) 185D and (g,h) 415D refer to different scales of magnifications for the corresponding sample.

Preparation of aerogels. Aerogels were synthesised from resorcinol and formaldehyde in presence of $\mathrm{Na}_{2} \mathrm{CO}_{3}$ as catalyst. The $\mathrm{pH}$ level of the starting solution is adapted at a neutral level with $\mathrm{HNO}_{3}$ and $\mathrm{NH}_{4} \mathrm{OH}$ buffers. The quantities of resorcinol (R), $\mathrm{Na}_{2} \mathrm{CO}_{3}$ catalyst (C), formaldehyde (F), and water (W) that were utilized in the preparation of the aerogel were 12.44 gram, $0.0240 \mathrm{~g}, 17.40 \mathrm{ml}$, and $32.60 \mathrm{ml}$, respectively. These quantities refer to molar ratios of $\mathrm{R}: \mathrm{F}=0.5, \mathrm{R}: \mathrm{C}=500$ and $\mathrm{R}: \mathrm{W}=0.05$. The medium temperature is controlled at $70 \pm 1{ }^{\circ} \mathrm{C}$. 

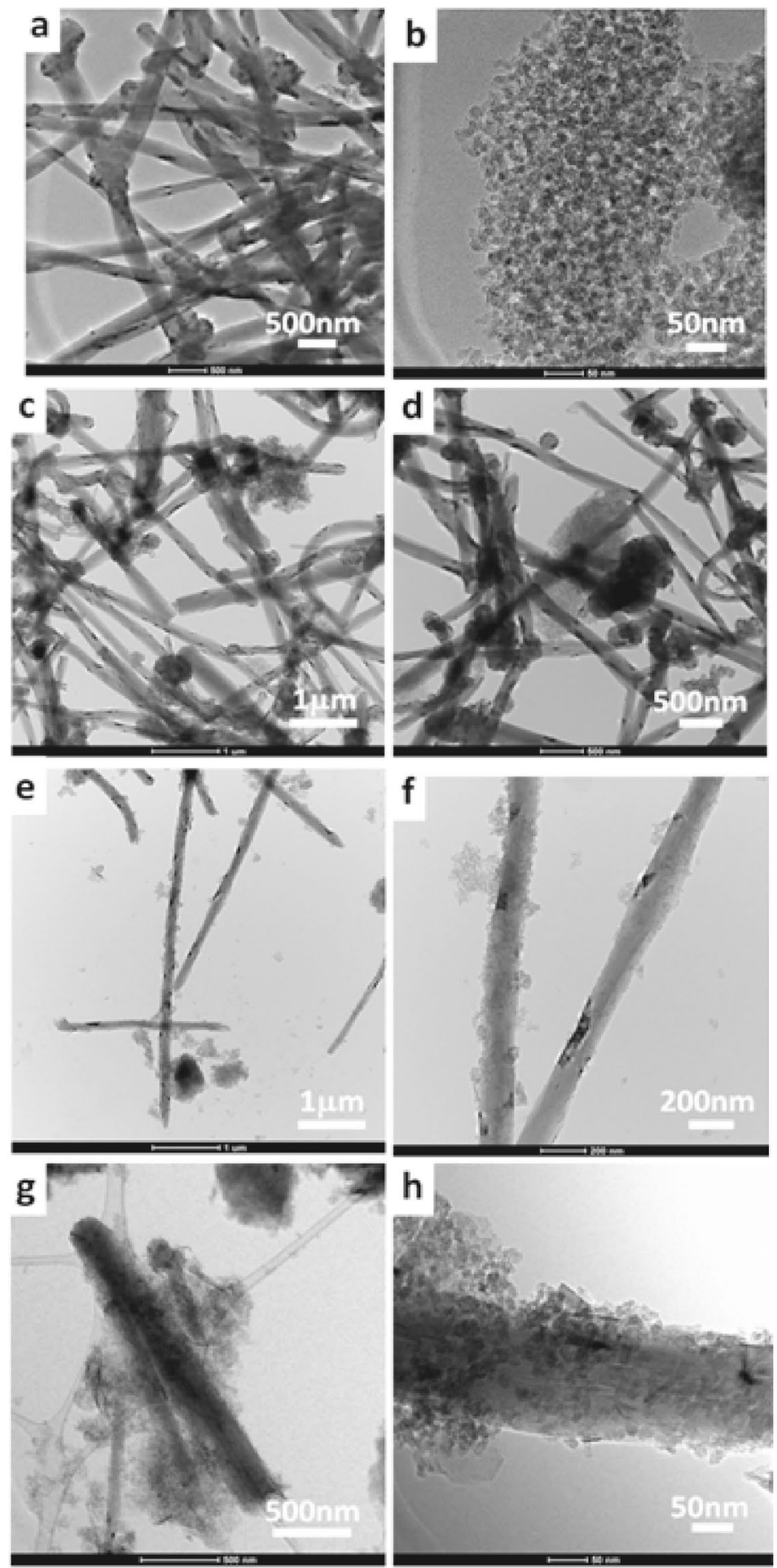

Figure 4. TEM photomicrographs of MWCNTs/RFA-CNp with 50/50 g/g at different aqueous mixing time of stirring and different scale of magnification. (a,b) refers to pristine MWCTs and CNp, respectively. Each couple of photomicrographs of (c,d) 0D, (e,f) 185D and (g,h) 415D refer to different scale of magnifications.

The gels were cured and dried by supercritical $\mathrm{CO}_{2}$ extraction as detailed elsewhere ${ }^{23,24}$. Supercritical drying is conducted by utilizing a critical point dryer (E3100 Critical Point Dryer, Quorum Technologies - Preparation for Excellence, UK).

Preparation of activated carbon aerogels. The dried resorcinol/formaldehyde aerogels are placed in a ceramic crucible into a tube furnace (Nabertherm $\mathrm{GmbH}$, Germany), while flowing a stream of nitrogen gas $\left(1.0 \times 10^{2} \mathrm{~cm}^{3} / \mathrm{min}\right)$. The tube furnace is firstly kept at ambient temperature for $1 / 2 \mathrm{~h}$ to make sure that air is fully replaced with nitrogen. Afterwards, the temperature of the furnace is increased up to $500^{\circ} \mathrm{C}$ with a rate of $10^{\circ} \mathrm{C} / \mathrm{min}$, 
kept at $500^{\circ} \mathrm{C}$ for $180 \mathrm{~min}$, and then let to cool down spontaneously to ambient temperature while flowing nitrogen. The outcome RF carbon aerogel is later activated within the same ceramic crucible (after cleaning thoroughly from previous residues) by switching the nitrogen gas with a carbon dioxide gas stream $\left(1.5 \times 10^{2} \mathrm{~cm}^{3} / \mathrm{min}\right)$, heating-up the sample again with a ramp of $10^{\circ} \mathrm{C} / \mathrm{min}$ up to $700^{\circ} \mathrm{C}$, keeping the sample at $700^{\circ} \mathrm{C}$ for $1 \mathrm{~h}$, and then cooling the product spontaneously to ambient temperature while still flowing $\mathrm{CO}_{2}$ gas $^{24,25}$. The outcome sample is redeemed to be an activated carbon aerogel and is called resorcinol-formaldehyde carbon aerogel nanoparticles, which are denoted as RFA-CNp.

Synthesis of carbon nanospheres-decorated carbon nanotubes. MWCNTs and RFA-CNp were mixed in a fixed weight proportion of 1:1 (this ratio is selected as a fair example of the amount used between the two main ingredients) in reflux with methanol while stirring for 0,185 and 415 days at $100^{\circ} \mathrm{C}$. The identity of these samples will be called hereafter; 0D, 185D and 415D, respectively. The sample 0D refers to mixing the samples manually in a dry state. All samples are then dried at $110^{\circ} \mathrm{C}$ for 3 days ${ }^{25}$.

\section{Characterization}

FT-Raman spectra were estimated by a Bruker FT-Raman spectrometer of type RFS 100/S. The morphological strutures of carbon materails were invetiagted via a FEI Nova ${ }^{\mathrm{TM}}$ nanoscanning electron microscope 450. Thermogravimetric analyses (TGA) are conducted (from $30{ }^{\circ} \mathrm{C}$ to $800^{\circ} \mathrm{C}$ with a heating rate of $10^{\circ} \mathrm{C} / \mathrm{min}$ ) utilizing a Perkin Elmer Pyris6 TGA analyzer with a flow of nitrogen. Transmission electron microscopy of (TEM) was conducted with a FEI Tecnai G2 F20 FE-TEM. X-ray diffraction (XRD) tests are carried out by Miniflex II Benchtop XRD apparatus, manufactured by Rigaku Corporation Japan.

Received: 4 December 2019; Accepted: 3 March 2020;

Published online: 17 March 2020

\section{References}

1. Moreno, D., Llorent-Martínez, E. J., Zougagh, M. \& Ríos, A. Decoration of multi-walled carbon nanotubes with metal nanoparticles in supercritical carbon dioxide medium as a novel approach for the modification of screen-printed electrodes. Talanta 161, 775-779 (2016).

2. Shi, L., Zhang, G. \& Wang, Y. Tailoring catalytic performance of carbon nanotubes confined $\mathrm{CuOeCeO} 2$ catalysts for $\mathrm{CO}$ preferential oxidation. Int. J. Hydrogen Energ. 43, 18211-18219 (2018).

3. Ihsanullah. Carbon nanotube membranes for water purification: Developments, challenges, and prospects for the future. Sep. Purif. Technol. 209, 307-337 (2019).

4. Xu, H., Li, L., Fan, G. \& Chu, X. DFT study of nanotubes as the drug delivery vehicles of Efavirenz. Computat. Theor. Chem. 113, 57-68 (2018).

5. Zheng, X., Su, Y., Chen, Y., Huang, H. \& Shen, Q. Global transcriptional responses of denitrifying bacteria to functionalized singlewalled carbon nanotubes revealed by weighted gene-coexpression network analysis. Sci. Total Environ. 613-614, 1240-1249 (2018).

6. Urper, O., Çakmak, I. \& Karatepe, N. Fabrication of carbon nanotube transparent conductive films by vacuum filtration method. Mater. Lett. 223, 210-214 (2018).

7. Jiao, Z., Wu, Q. \& Qiu, J. Preparation and electrochemical performance of hollow activated carbon fiber-Carbon nanotubes threedimensional self-supported electrode for supercapacitor. Mater. Design. 154, 239-245 (2018).

8. Shi, Y. et al. Carbon nanotube decorated with silver nanoparticles via noncovalent interaction for a novel nonenzymatic sensor towards hydrogen peroxide reduction. J. Electroanal. Chem. 656, 29-33 (2011).

9. Ye, X. R., Lin, Y. \& Wai, C. M. Decorating catalytic palladium nanoparticles on carbon nanotubes in supercritical carbon dioxide. Chem. Commun. 9, 642-643 (2003).

10. Katz, E. \& Willner, I. Biomolecule-functionalized carbon nanotubes: Applications in nanobioelectronics. Chem. Phys. Chem. 5, 1084-104 (2004)

11. Oschatz, M. et al. Carbide-derived carbon aerogels with tunable pore structure as versatile electrode material in high power supercapacitors. Carbon 113, 283-291 (2017).

12. Yang, X., Wei, C. \& Zhang, G. Activated carbon aerogels with developed mesoporosity as high-rate anodes in lithium-ion batteries. J. Mater. Sci. 51, 5565-5571 (2016).

13. Sarapuu, A. et al. Electrocatalysis of oxygen reduction by iron-containing nitrogen-doped carbon aerogels in alkaline solution. Electrochim. Acta 230, 81-88 (2017).

14. Bi, H. et al. New graphene form of nanoporous monolith for excellent energy storage. Nano Lett. 16, 349-354 (2016).

15. Xu, Y., Ren, B., Wang, S., Zhang, L. \& Liu, Z. Carbon aerogel-based supercapacitors modified by hummers oxidation method. J. Colloid Interface Sci. 527, 25-32 (2018).

16. Zhang, K., Ang, B. T., Zhang, L. L., Zhao, X. S. \& Wu, J. Pyrolyzed graphene oxide/resorcinol-formaldehyde resin composites as high-performance supercapacitor electrodes. J. Mater. Chem. 21, 2663-2670 (2011).

17. Yahya, N., Rehman, Z. U. \& Shafie, A. MWCNT for ambient urea synthesis. Physica B. 545, 358-369 (2018).

18. Bokobza, L., Bruneel, J.-L. \& Couzi, M. Raman spectra of carbon-based materials (from graphite to carbon black) and of some silicone composites. C. 1, 77-94 (2015).

19. Zhang, W.-J., Bagreev, A. \& Rasouli, F. Reaction of $\mathrm{NO}_{2}$ with activated carbon at ambient temperature. Ind. Eng. Chem. Res. 47, 4358-4362 (2008).

20. Sun, Y., Li, C. \& Zhang, A. Preparation of Ni/CNTs catalyst with high reducibility and their superior catalytic performance in benzene hydrogenation. Appl. Catal. A Gen. 522, 180-187 (2016).

21. Reddy, A. L. M. \& Ramaprabhu, S. Hydrogen storage properties of nanocrystalline Pt dispersed multi-walled carbon nanotubes. Int. J. Hydrogen Energ. 32, 3998-4004 (2007).

22. Vellingiri, L., Annamalai, K., Kandasamy, R. \& Kombiah, I. Characterization and hydrogen storage properties of SnO2 functionalized MWCNT nanocomposites. Int. J. Hydrogen Energ. 43, 10396-10409 (2018).

23. Pérez-Caballero, F., Peikolainen, A.-L. \& Koel, M. Preparation of nanostructured carbon materials. P. E.S.T. Acad. Sci. 57, 48-53 (2008).

24. Awadallah-F, A., Elkhatat, A. M. \& Al-Muhtaseb, S. A. Impact of synthesis conditions on meso-and macropore structures of resorcinol-formaldehyde xerogels. J. Mater. Sci. 46, 7760-7769 (2011).

25. Awadallah-F, A. \& Al-Muhtaseb, S. A. Carbon nanospheres-decorated carbon nanotubes. PCT Application No. PCT/IB2019/051690 filed March 2 (2019). 


\section{Acknowledgements}

This publication was made possible by the NPRP award (NPRP 08-014-2-003) from the Qatar National Research Fund (a member of The Qatar Foundation). The statements made herein are solely the responsibility of the authors. Technical support from the Department of Chemical Engineering, the Central Laboratories Unit (CLU) and Gas Processing Centre (GPC) at Qatar University is also acknowledged. The publication of this article was funded by the Qatar National Library.

\section{Author contributions}

Conceptualization, A.A.-F. and S.A.A.-M.; methodology, A.A.-F. and S.A.A.-M.; validation, A.A.-F. and S.A.A.-M.; formal analysis, A.A.-F.; investigation, A.A.-F.; resources, S.A.A.-M.; data curation, A.A.-F.; writingoriginal draft preparation, A.A.-F.; writing-review and editing, S.A.A.-M.; visualization, A.A.-F. and S.A.A.-M.; supervision, S.A.A.-M.; project administration, S.A.A.-M.; funding acquisition, S.A.A.-M.

\section{Competing interests}

The authors declare no competing interests.

\section{Additional information}

Correspondence and requests for materials should be addressed to S.A.-M.

Reprints and permissions information is available at www.nature.com/reprints.

Publisher's note Springer Nature remains neutral with regard to jurisdictional claims in published maps and institutional affiliations.

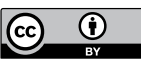

Open Access This article is licensed under a Creative Commons Attribution 4.0 International License, which permits use, sharing, adaptation, distribution and reproduction in any medium or format, as long as you give appropriate credit to the original author(s) and the source, provide a link to the Creative Commons license, and indicate if changes were made. The images or other third party material in this article are included in the article's Creative Commons license, unless indicated otherwise in a credit line to the material. If material is not included in the article's Creative Commons license and your intended use is not permitted by statutory regulation or exceeds the permitted use, you will need to obtain permission directly from the copyright holder. To view a copy of this license, visit http://creativecommons.org/licenses/by/4.0/.

(c) The Author(s) 2020 